\title{
TiO and VO broad band absorption features in the optical spectrum of the atmosphere of the hot-Jupiter HD 209458b
}

\author{
J.-M. Désert ${ }^{1}$, A. Vidal-Madjar ${ }^{1}$, A. Lecavelier des Etangs ${ }^{1}$, D. Sing $^{1}$, D. Ehrenreich ${ }^{2}$, G. Hébrard ${ }^{1}$, and R. Ferlet ${ }^{1}$ \\ ${ }^{1}$ Institut d'Astrophysique de Paris, CNRS (UMR 7095) - Université Pierre \& Marie Curie, 98 bis boulevard Arago \\ 75014 Paris, France \\ e-mail: desert@iap.fr \\ 2 Laboratoire d'astrophysique de l'observatoire de Grenoble, Université Joseph Fourier, CNRS (UMR 5571), \\ BP 53, 38041 Grenoble Cedex 9, France
}

Received 9 June 2008 / Accepted 3 September 2008

\section{ABSTRACT}

\begin{abstract}
Aims. The presence of titanium oxide ( $\mathrm{TiO})$ and vanadium oxide (VO) gas phase species is searched for in the atmosphere of the hot Jupiter HD 209458b.

Methods. We compared a model for the planet's transmitted spectrum to multi-wavelength eclipse-depth measurements (from 3000 to $10000 \AA$ ) using archived HST-STIS time series spectra. We make use of these observations to search for spectral signatures from extra absorbers in the planet atmosphere between 6000 and $8000 \AA$.

Results. Along with sodium depletion and Rayleigh scattering recently published for this exoplanet atmosphere, an extra absorber of uncertain origin, redward of the sodium lines, is present in the atmosphere of the planet. Furthermore, this planet has a stratosphere experiencing a thermal inversion caused by the capture of optical stellar flux by absorbers at altitude. Recent models have predicted that the presence of $\mathrm{TiO}$ and $\mathrm{VO}$ in the atmosphere of $\mathrm{HD} 209458 \mathrm{~b}$ may be responsible for this temperature inversion. Although no specific $\mathrm{TiO}$ and $\mathrm{VO}$ spectral band head signatures have been identified unambiguously in the observed spectrum, we suggest here that the opacities of those molecules are possible candidates to explain the remaining continuous broad band absorption observed between 6200 and $8000 \AA$. To match the data reasonably well, the abundances of TiO and VO molecules are evaluated from ten to one thousand times below solar. This upper limit result is in agreement with expected variations with altitude due to depletion effects such as condensation.
\end{abstract}

Key words. publications - bibliography

\section{Introduction}

The discovery of transiting extrasolar giant planets (EGP) has been followed by direct detections and characterization of their atmospheres. Because of the wavelength-dependent opacities of absorbing species, measurement of relative changes in eclipse depth as a function of wavelength during primary transit has the potential to reveal the presence (or absence) of specific chemical species (Seager \& Sasselov 2000; Hubbard et al. 2001; Brown 2001). In the case of HD 209458b's atmosphere, transmission spectroscopy led to the detection of sodium (Charbonneau et al. 2002). The small size of the atmospheric sodium signature has made its detection from ground-based telescopes difficult. Only recently, Redfield et al. (2008) and Snellen et al. (2008) reported the detection of $\mathrm{NaI}$ in the atmosphere of HD 189733b and HD 209458b, respectively. Absorptions of several percent for H I Lyman- $\alpha$, O I and C II have been measured in the hydrodynamically escaping upper atmosphere (Vidal-Madjar et al. 2003, 2004, 2008). More recently, Rayleigh scattering by $\mathrm{H}_{2}$ molecules has been identified (Lecavelier et al. 2008b) and a temperature-pressure (TP) profile with inversion was derived (Sing et al. 2008b). This temperature inversion leads to high temperatures at both low and high pressure. Note that this temperature bifurcation was very well predicted by atmospheric models of strongly irradiated planets (Hubeny et al. 2003). In the lower part of the atmosphere ( $\sim 30 \mathrm{mbar}$ ), the temperature is found to be in the range 1900-2400 K (Lecavelier et al. 2008b), which corresponds to the M/L/T brown dwarf regime, as expected for a hot Jupiter such as HD 209458b (Kirkpatrick 2005).

In the cool atmosphere of sub-stellar objects like brown dwarfs and close-in EGPs, the strongest absorption features are expected to be those of alkali metals like sodium and potassium (Seager \& Sasselov 2000; Sudarsky et al. 2000; Burrows et al. 2000). This is due to the condensation and precipitation of elements in the atmosphere which clears the atmosphere of most of the metals and keeps the less refractory alkali metals (Burrows \& Sharp 1999; Lodders 1999; Burrows et al. 2002; Sudarsky et al. 2003). Thus, in contrary to the spectrum of HD 189733b (Pont et al. 2007; Lecavelier des Etangs et al. 2008a), the spectrum of HD 209458b should be dominated by absorption from sodium $\mathrm{Na}$ I and potassium $\mathrm{K}$ I.

However, depending on the effective temperature, a large number of diatomic and polyatomic molecules are predicted to be present according to various models of brown dwarfs and hot Jupiters (Burrows \& Sharp 1999; Lodders 1999; Allard et al. 2001; Lodders 2002; Hubeny et al. 2003; Burrows et al. 2006). Among those molecules and at a temperature above $1800 \mathrm{~K}$, titanium oxide ( $\mathrm{TiO}$ ) and vanadium oxide (VO) in gas phase equilibrium are most probably present with a high abundance in strongly irradiated planet atmospheres (Seager et al. 1998; Hubeny et al. 2003; Fortney et al. 2007). Furthermore, the low albedo measurements of HD 209458b (Rowe et al. 2006) rule 
out most of the absorbents, except $\mathrm{TiO}$ and VO. Recently, the interpretation of visible observations obtained during the transit of this planet (Sing et al. 2008a), as well as the interpretation of near infrared observations taken during its secondary eclipse (Knutson et al. 2008) require that HD 209458b experience an inversion in the atmospheric $P-T$ profile and a have stratosphere (Sing et al. 2008b; Burrows et al. 2007c; Burrows et al. 2008). Such an inversion could be due to the absorption of the incident visible light by $\mathrm{TiO}$ and $\mathrm{VO}$ molecules (Fortney et al. 2007). Ongoing models (Burrows et al. 2008) claim that this inversion must be caused by the capture of the incident optical stellar flux by a stratospheric absorber that could potentially be TiO and VO. Similarly, other studies (Hubeny et al. 2003; Fortney et al. 2007) have more particularly investigated the effects of $\mathrm{TiO}$ and $\mathrm{VO}$ which absorb much of the incoming stellar radiation high in the atmospheres of the hottest CEGPs.

In this work, we use STIS spectra obtained during planetary transits at two spectral resolutions (low and medium) to search for direct evidence of the presence of $\mathrm{TiO}$ and $\mathrm{VO}$ in the planet's atmosphere. Both datasets are combined to extend the measurements over the entire optical regime to quantify other possible absorbers appearing in the transmission spectrum. Rayleigh scattering and sodium absorption have been proposed to explain the spectral features observed between $3000 \AA$ and $6000 \AA$ (Lecavelier des Etangs et al. 2008b; Sing et al. 2008a,b) but, additional absorptions from $6100 \AA$ up to $8000 \AA$ remain unattributed. These absorptions cannot be due to optically thick high altitude clouds, which would otherwise mask the detected signature of Rayleigh scattering (Lecavelier des Etangs et al. 2008b). Since TiO and VO molecules are expected to be present in high abundance for the range of temperatures and pressures for strongly irradiated hot Jupiters, their opacities could largely contribute to the observed absorption in this wavelength domain (Seager et al. 1998; Burrows \& Sharp 1999; Sharp \& Burrows 2007).

After a brief description of the observations and interpretations (Sect. 2), we present, in Sect. 3, the model together with the method used to calculate the atomic and molecular opacities. Finally, we estimate the contribution of TiO and VO to the observed transmission spectrum for several abundance scenarios (Sect. 4) and discuss the consequences for the atmospheric chemistry and aeronomy of HD 209458b (Sect. 5).

\section{Observations}

The HST-STIS G750L and G430L low resolution grating observations of HD 209458b analyzed here are also detailed in Knutson et al. (2007a), Ballester et al. (2007) and Sing et al. (2008a). For both the G750L and G430L gratings, two visits were performed for each grating, of five consecutive orbits each. Each visit spans one completed transit of HD 209458b in 2003. Together they cover the combined range 2900-10300 A, with some overlap around $5300 \AA$ with a resolving power $R=500$. While the broad-band spectrophotometric analysis of Knutson et al. (2007a) was used to identify water absorption features in the atmosphere of HD 209458b at wavelengths greater than $9000 \AA$ (Barman 2007), here we analyze the spectrum at full resolution.

We directly use HD 209458b absorption depths (AD, see definition in Sect. 3.3) between $4000 \AA$ and $8000 \AA$ provided by Sing et al. (2008a). The low resolution transit spectrum ratio, limb darkening corrected, is plotted in Fig. 1. The spectrum below $8000 \AA$ is considered here where the absorption due to water molecules is negligible. Three prominent broadband absorption features are revealed. The near ultraviolet (NUV) absorption, at wavelength $\lambda \leq 5000 \AA$, was first reported by Ballester et al. (2007) and explained by the absorption of a hot hydrogen layer within the atmosphere. Lecavelier des Etangs et al. (2008b) propose an alternative explanation invoking the Rayleigh scattering by $\mathrm{H}_{2}$ molecules. Within the same datasets, Sing et al. (2008b) found that the Na spectral line profile is characterized by a wide absorption with a sharp transition to a narrow absorption profile at higher altitudes values. This sharp transition is interpreted by condensation or ionization which deplete $\mathrm{Na}$ atoms in the upper atmosphere. Using a global fit to these data, from $3000 \AA$ to $6200 \AA$, Sing et al. (2008b) determine the average pressuretemperature profile $(P-T)$ at the planetary terminator. Two types of $P-T$ profile were derived which both can lead to the depletion of Na I atoms. One profile implies the condensation of $\mathrm{Na}$ I and the other the ionization of Na I. We use the former (Fig. 2). Both profiles experience hot temperatures, above $1800 \mathrm{~K}$ at $50 \mathrm{mbar}$, due to Rayleigh scattering (Lecavelier et al. 2008b). At such a temperature, $\mathrm{TiO}$ and $\mathrm{VO}$ are in the gas phase.

A third broad absorption feature in the 6200-8000 $\AA$ range appears on the AD spectrum and is still unexplained. This absorption is composed of two remarkable features visible in Fig. 1:

1. a strong and broad spectral absorption feature centered at about $6250 \AA$ which is slightly smaller than for the Na I $(A D=0.0148 \%$ over $100 \AA)$. Other spectral signatures appearing at higher wavelengths are weaker than the $6250 \AA$ one;

2. a broad and continuous absorption level above $7000 \AA$, systematically higher than the lowest observational AD $(0.0146 \%$ over $1500 \AA)$. This broad continuous flat absorption excess is weaker than the spectral feature at $6250 \AA$ but is equally important since it covers a higher spectral domain, and has a much larger absorption level than predicted by extrapolating Rayleigh scattering and $\mathrm{Na}$ I or K I broad wings.

In Fig. 1, we overplotted the radii presented by Knutson et al. (2008) that were derived using a spectrophotometric bandpass across roughly $1000 \AA$ bins. These radii are found to be slightly different to those presented in this paper, especially in the region of interest of this study (over $6000 \AA$ ), though the differences are either close to or within the error bars of the two studies. This differences could be explained by the supplementary corrections in the data treatement employed by Sing et al. (2008) who corrected the transmission spectrum for telluric contamination and various systematics, quantified the level of systematic red noise to take it into account in the computed errors and finally applied a spectral limb darkening correction before binning. Note that the systematic errors have a weak wavelength dependance, affecting the absorption depth values over large wavelength regions in a similar manner, largely preserving the wavelength structure of narrow bands such as the $6250 \AA$ feature.

Looking at the low resolution spectrum, we find the minimum AD of $0.01453 \%$ around $5000 \AA$, which indicates a base pressure of 33 mbar in the $P-T$ profile (Lecavelier des Etangs et al. 2008b). Conversely, the maximum AD of $0.0149 \%$ is obtained for the central pixel of the $\mathrm{Na}$ I line which corresponds to a pressure of 1 mbar (Sing et al. 2008b).

Below we present possible identifications of absorbers that can simultaneously explain the spectral features between 6200 


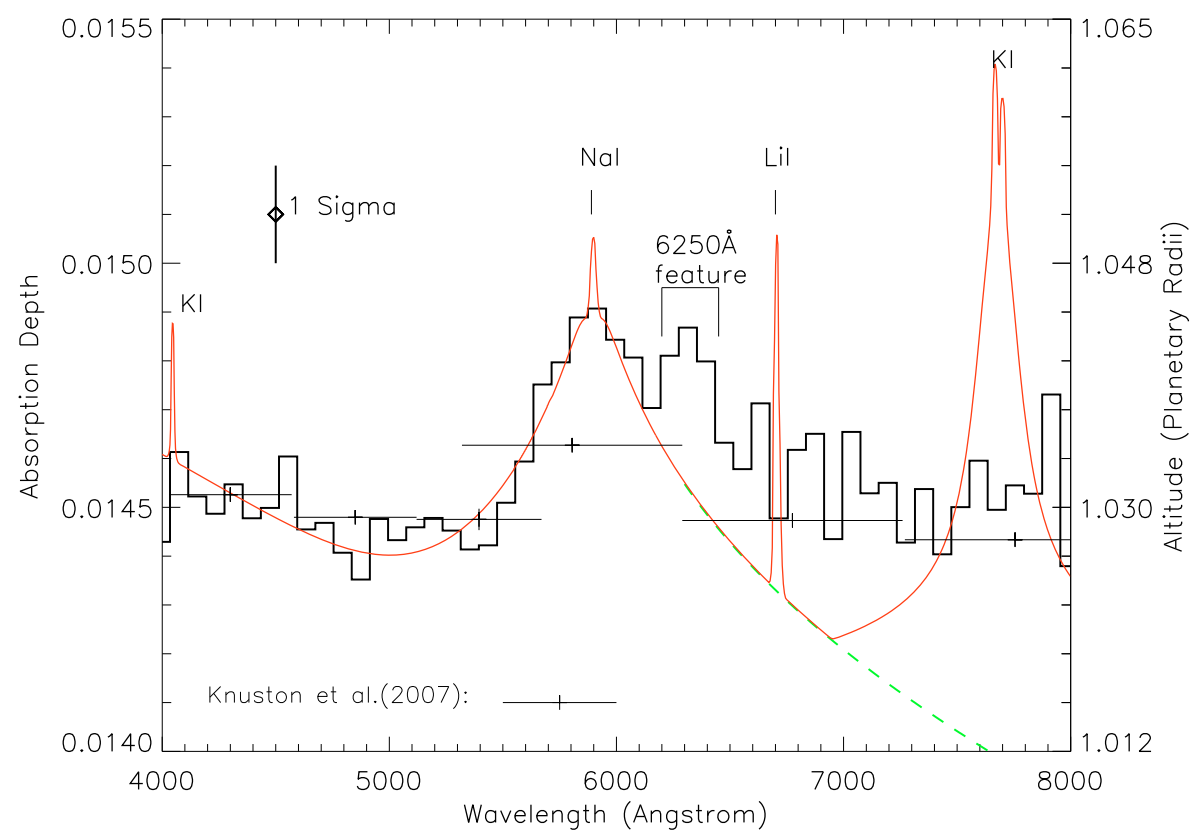

Fig. 1. The low resolution STIS measurements of the planetary transit absorption depth (AD) corrected from limb-darkenning effects and binned by 60 pixels (histogram). The observed $1 \sigma$ errorbar is plotted above the spectrum. The radii derived by Knutson et al. (2007) with the corresponding error bars and spectrale bins are plotted (squares). The dashed line corresponds to the best fit model assuming Rayleigh scattering and sodium absorption with the physical $T-P$ profile plotted in Fig. 2 . The difference between the dashed curve and the observed AD spectrum for wavelengths over $6000 \AA$ indicates that remaning absorbants should be presents in this spectral domain. Overplotted with the continuous line is the same model, with atomic lithium and potassium with solar abundances. This model cannot reproduce the observations above $6200 \AA$.

and $7000 \AA$ and the broad relatively flat absorption excess from 7000 to $8000 \AA$.

\section{Model description}

In order to interpret the transit observations, we developed a model that calculates the absorptions of atomic and molecular lines in the atmosphere of a transiting planet.

Transit spectroscopy probes the transition region between the day and night sides, called the limb. We used the geometry of a transiting system (Brown 2001) and a model originally developed by Ehrenreich et al. (2006) and adapted for this work. Along a single cord, stellar photons cross several levels of the spherically stratified atmosphere of the planetary limb.

The total opacity, $\tau_{\lambda}$, along a cord (parallel to the line of sight) as a function of its impact parameter $b$ and the altitude $h$, is given as:

$\tau_{\lambda, i}(b)=2 \int_{0}^{+\infty} \sigma_{\lambda, i} n_{i}(h) \mathrm{d} l$,

where $\sigma_{\lambda, i}$ is the cross section coefficient for the species $i$ at the wavelength $\lambda$, and $n_{i}(h)$ is the density of the species $i$ at an altitude $h$ in the atmosphere (see Fig. 1 in Ehrenreich et al. 2006).

\subsection{Atomic and molecular line opacities}

In the case of alkali atoms, we calculate the opacity using the oscillator strength, the radiative lifetime and the collisional broadening taken from Morton et al. (1991) and Iro et al. (2005) who used Burrows et al. (2000) to determine the line shapes. Further progress has been made in the theory of these profiles (Burrows et al. 2003; Allard et al. 2005). The difference between these new line shapes and the ones used in this study resides mainly in the far wings. However, the contribution of other absorbers dominates the spectrum in the far wings of the alkali lines. Thus, we found that the calculations of Morton et al. (1991) and Iro et al. (2005) were precise enough for our study.

In the case of molecules, we calculate the line strengths for each line of each species using the available data. We derive the strength at local thermal equilibrium (LTE), denoted $S$, of the molecular spectral line from the general equation (Sharp \& Burrows 2007):

$S=\frac{\pi e^{2} g_{i} f_{i j}}{m_{e} c} \frac{\mathrm{e}^{-h c F_{i} / k T}}{Q(T)}\left[1-\mathrm{e}^{-h c\left(F_{j}-F_{i}\right) / k T}\right]$,

where $g_{i}$ and $f_{i j}$ are the statistical weight of the $i$ th energy level and the oscillator strength for a transition from that level to a higher level $j$. The excitation energies $F_{i}$, and $F_{j}$ are the term values in $\mathrm{cm}^{-1}$ of the $i$ th and $j$ th levels participating in the transition. In addition, $Q(T)$ is the partition function of the species at some temperature $T$. We compute the partition function as described by Sauval \& Tatum (1984), considering a fourth degree polynomial function of $\log \left(\frac{5040}{T}\right)$. The molecular lines are broadened using a Voigt profile. At pressure $P$, the collisional broadening is calculated using (Sharp \& Burrows 2007):

$\Delta v_{\text {Lorentz }}=w_{\mathrm{o}} P$

with $w_{\mathrm{o}}=0.1 \mathrm{~cm}^{-1} \mathrm{bar}^{-1}$.

Finally, we obtain the total monochromatic opacity of the atmosphere by summing the individual contributions for each atom and molecule weighted by their respective abundances at each altitude level.

\subsection{Grid of opacities}

Computing the opacities directly at each altitude with the corresponding temperature and pressure would require intense calculation. Instead, we precalculate a grid of opacities for each molecule. Our grid is composed of 42 cells with a range of temperature from 100 to $5000 \mathrm{~K}$ and a range of pressure from 1 to $10^{-6}$ bar. Opacities have been computed from 2900 to $10000 \AA$ with a resolution of $0.005 \AA$ which corresponds to 5 million elements per cell. For a given $P-T$ profile we then calculate the corresponding opacities by applying a linear interpolation on the grid cells.

\subsection{Absorption depth (AD)}

The output of the model is a spectrum ratio defined by Brown (2001) as the ratio of the flux received during the transit to 


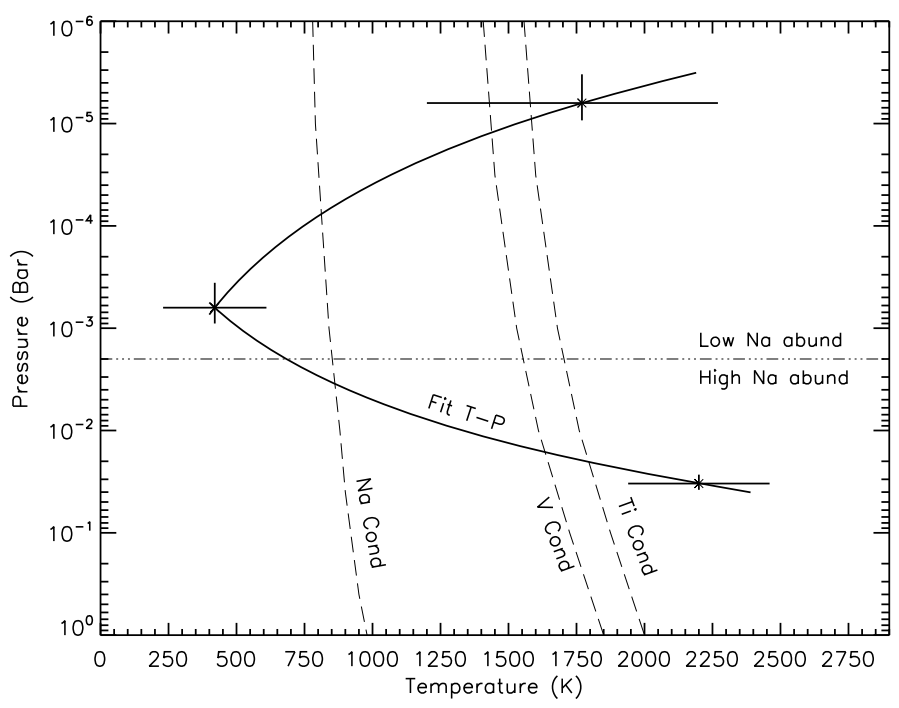

Fig. 2. The atmospheric temperature-pressure profile ( $P-T$ profile) with error bars derived by Sing et al. (2008b) used in this study. The dashed lines correspond to the condensation curves for titanium, vanadium and sodium. The three points of this $T-P$ profile are derived from the fit of the observed absorption depth curve. The hot point at a pressure of 0.05 bar is imposed by the Rayleigh scattering (see Lecavelier et al. 2008).

the flux received when the planet is not occulting the star of radius $R_{\star}$. Otherwise indicated by the absorption depth (AD), it is the occultation of the surface of the star by the surface of the planet at each wavelength. The surface of the planet is twofold: the optically thick disk of radius $R_{\mathrm{P}}$ and the wavelengthdependent occultation by the atmosphere that surrounds the planetary disk expressed as an equivalent surface. We used $R_{\mathrm{P}}=1.32 R_{\text {jup }}$ and $R_{\star}=1.125 R_{\odot}$ from Knutson et al. (2007a). Lecavelier des Etangs et al. (2008b) show that the observed AD is well approximated by:

$\mathrm{AD}_{\lambda}=\mathrm{AD}_{0}\left(1+\frac{2 H}{R_{\mathrm{P}}} \ln \frac{\sigma_{\lambda}}{\sigma_{\lambda_{0}}}\right)$

where $\mathrm{AD}_{0}$ is $\mathrm{AD}$ at $\lambda=\lambda_{0}$ and $H$ the scale height. Thus the observed mean $\mathrm{AD}$ over a wavelength range of a given spectral element (typically $50 \AA$ ) is proportional to the mean of the logarithm of the cross section. Therefore, we calculate the effective cross section in a given spectral element by averaging the logarithm of the cross section calculated at a much higher resolution:

$\sigma_{\text {bin }}\left[\lambda_{i} ; \lambda_{j}\right]=\exp \left(\int_{\lambda_{i}}^{\lambda_{j}} \frac{\ln \sigma_{\lambda}}{\lambda_{i}-\lambda_{j}} \mathrm{~d}_{\lambda}\right)$

\section{Analysis}

Spectral signatures, line profiles and abundances vary depending on the temperature and pressure vertical profile in the atmosphere. Here we use the vertical $P-T$ profile presented in Fig. 2 and two sodium mixing ratios from Sing et al. (2008b) as well as the Rayleigh scattering derived by Lecavelier des Etangs et al. (2008b). The AD of $0.01453 \%$, corresponding to a pressure of 33 mbar, is taken as the reference at 1.32 Jupiter radii (Knutson et al. 2007). Using those hypotheses, we calculate the absorption spectrum with only the abundances as free parameters. We vary the abundances to find the best fit to the data using a $\chi^{2}$ minimization between the data and the model.
Table 1. List of the most probable species with their corresponding solar abundances which have significant absorptions in the 6000-8000 $\AA$ wavelength domain (from Lodders 2002; Sharp \& Burrows 2007). This list is ordered by the product of an average of the cross section between 4000 and $8000 \AA$ and its corresponding solar abundance. This ordered list gives a rough estimate of the plausible detectability for each atomic and molecular species.

\begin{tabular}{cccc}
\hline \hline Element & $\chi_{\odot}$ & $\left\langle\sigma_{\lambda}\right\rangle\left(\mathrm{cm}^{2}\right)$ & $\chi_{\odot}\left\langle\sigma_{\lambda}\right\rangle\left(\mathrm{cm}^{2}\right)$ \\
\hline $\mathrm{Na}$ & $3 . e-6$ & $1 . e-19$ & $1 . \mathrm{e}-25$ \\
$\mathrm{~K} \mathrm{I}$ & $2 . \mathrm{e}-7$ & $1 . \mathrm{e}-20$ & $1 . \mathrm{e}-27$ \\
$\mathrm{Li} \mathrm{I}$ & $1 . \mathrm{e}-9$ & $1 . \mathrm{e}-19$ & $1 . \mathrm{e}-28$ \\
\hline $\mathrm{SiO}$ & $1 . \mathrm{e}-5$ & $1 . \mathrm{e}-17$ & $1 . \mathrm{e}-22$ \\
$\mathrm{TiO}$ & $1 . \mathrm{e}-7$ & $1 . \mathrm{e}-16$ & $1 . \mathrm{e}-23$ \\
$\mathrm{VO}$ & $1 . \mathrm{e}-8$ & $1 . \mathrm{e}-16$ & $1 . \mathrm{e}-24$ \\
$\mathrm{MgH}$ & $1 . \mathrm{e}-9$ & $1 . \mathrm{e}-17$ & $1 . \mathrm{e}-26$ \\
$\mathrm{H}_{2} \mathrm{O}$ & $1 . \mathrm{e}-3$ & $1 . \mathrm{e}-24$ & $1 . \mathrm{e}-27$ \\
$\mathrm{FeH}$ & $1 . \mathrm{H}-9$ & $1 . \mathrm{e}-19$ & $1 . \mathrm{e}-28$ \\
$\mathrm{CaH}$ & $1 . \mathrm{e}-11$ & $1 . \mathrm{e}-17$ & $1 . \mathrm{e}-28$ \\
$\mathrm{CrH}$ & $1 . \mathrm{e}-10$ & $1 . \mathrm{e}-19$ & $1 . \mathrm{e}-29$ \\
\hline
\end{tabular}

\subsection{Atomic line}

The most important absorptions by atomic lines are those of alkali metals, Na I, KI and Li I (see Table 1). This table provides the lines with the strongest abundance weighted cross-section in the considered wavelength range. None of these atomic lines, nor $\mathrm{H} \alpha$, can explain these observed spectral feature at $6250 \AA$.

In the low resolution transit spectrum, we do not detect significant absorption in the K I line at $7698 \AA$ A nor in the Li I doublet at $6708 \AA$. These non detections exclude the possibility of solar abundance over the whole atmosphere for these two species (see Fig. 1). Assuming a constant mixing ratio, we constrain the $\mathrm{KI}$ and $\mathrm{Li}$ I abundances to be lower than $2 \times 10^{-3}$ and $2 \times 10^{-1}$ solar, respectively $(1 \sigma)$. Of course, these upper limits do not exclude higher abundances below the altitude corresponding to the observed absorption depth of $\sim 0.0145 \%$.

We conclude that the absorption depth measured between 6200 and $8000 \AA$ cannot be explained by the strong absorption lines from abundant atomic species. The line profile of $\mathrm{Na}$ I does not affect the results on $\mathrm{TiO} / \mathrm{VO}$ abundances since the remaining broad band absorption begins $200 \AA$ away from the center of the line on the red side. In the following, we consider possible absorptions by molecules.

\subsection{Molecular line}

A list of plausible molecular species absorbing in the $6000 \AA$ region is presented in Table 1.

Although the molecules $\mathrm{CH}_{4}, \mathrm{NH}_{3}$ and $\mathrm{CO}$ are the most important source of opacities in the infrared, their effects are negligible in the visible wavelength domain; they are not considered in the following analysis.

Using the abundance weighted mean cross-section list, we found that the most plausible species are $\mathrm{SiO}, \mathrm{TiO}$ and $\mathrm{VO}$ respectively. Silicon oxide ( $\mathrm{SiO}$ ) absorption mainly occurs below $2000 \AA$, outside our wavelength domain. As a result, TiO and VO are predicted to be the most abundant and the most important sources of opacity in the present domain (Fortney et al. 2006b; Lodders 2002). However, since both species condense, their abundances decrease below $1500 \mathrm{~K}$.

Amongst all the other molecular absorbers that can be used to explain the observation in this wavelength range, the hydride metals $\mathrm{CrH}, \mathrm{FeH}, \mathrm{MgH}$, and $\mathrm{CaH}$ were considered as potential 


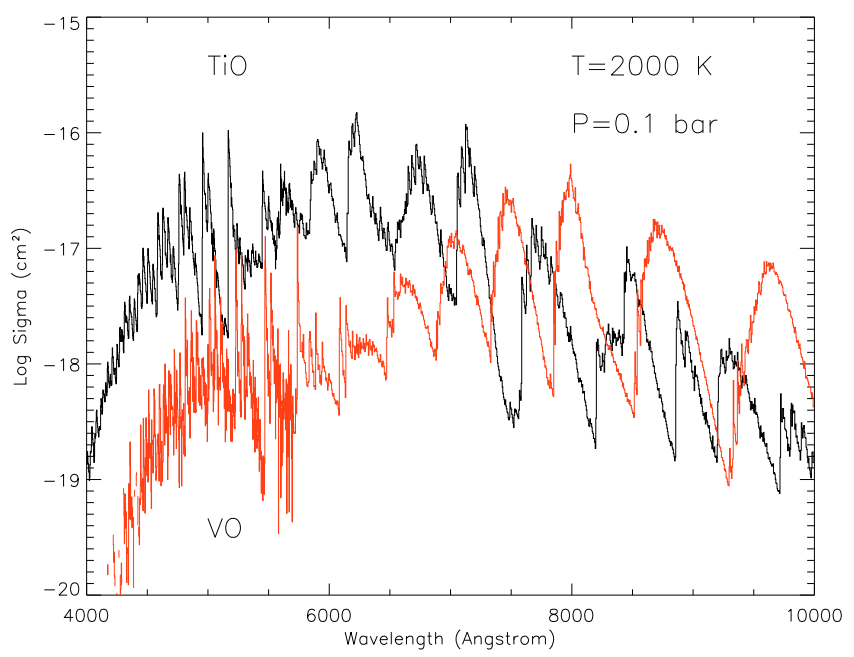

Fig. 3. The $\log _{10}$ of the monochromatic cross-section $\sigma\left(\mathrm{cm}^{2}\right)$ as a function of wavelength for the vibration-rotation transitions of $\mathrm{TiO}$ and $\mathrm{VO}$. The contribution due to different isotopes is included. TiO has a strong absorption feature shortward of $7500 \AA$, and has a strong peak near $6200 \AA$ As observed in Fig. 1 .

candidates to explain the shape of the spectrum. However, the abundance of those elements drops with decreasing temperature below $2000 \mathrm{~K}$, due to the formation of condensates (Lodders \& Fegley 2006; Kirkpatrick 2005). Furthermore, their absorption domain does not match the one considered here, e.g. $\mathrm{H}_{2} \mathrm{O}$.

Among plausible molecules in the atmosphere of HD 209458b, TiO and VO have the largest cross-section in the visible domain. We explore the extent to which various abundances of $\mathrm{TiO}$ and $\mathrm{VO}$ can be used to reproduce both the medium and low resolution spectra. We derived the LTE strength $S$ (Eq. (2)) of the molecular spectral lines from Plez (1998). These data list about 3 million lines from 4000 to nearly $30000 \mathrm{~cm}^{-1}$, covering the whole visible part of the spectrum for the transitions of the most abundant isotopes ${ }^{48} \mathrm{TiO}$, together with ${ }^{46} \mathrm{TiO},{ }^{47} \mathrm{TiO},{ }^{49} \mathrm{TiO}$, and ${ }^{50} \mathrm{TiO}$, for nine different electronic band systems. Using the grid previously described in Sect. 3.2, we compute theoretical AD including $\mathrm{TiO}$ and VO absorption at each wavelength (see Fig. 3). At a temperature of $2000 \mathrm{~K}$ and a pressure of $0.1 \mathrm{bar}$, the lines are significantly broadened, thus the rapid fluctuations in cross-section over short wavelength intervals are suppressed, revealing the main band features. At significantly lower pressures, the broadening of the lines is much smaller, the cross-section shows rapid variations as a function of wavelength and the broad main features do not show up so clearly.

In the following sections, we explore different scenarios for the atmospheric abundance of $\mathrm{TiO} / \mathrm{VO}$ in order to adjust our model to the observations. We fit the data with an atmospheric model including $\mathrm{Na}$ I, Rayleigh scattering, and TiO and/or VO in different abundances. In a first step, we consider a single abundance of $\mathrm{TiO} / \mathrm{VO}$ over the whole atmosphere. In a second step, we will assume two levels of abundance of $\mathrm{TiO} / \mathrm{VO}$. We finally discuss the fits obtained using those different abundances.

\subsection{TiO}

As a first step, we consider only $\mathrm{TiO}$ and constrain the abundance by fitting the observational low resolution spectrum ratio. Starting with the simplest assumption, we consider a solar abundance over the whole atmosphere of the planet.
Our model gives an AD average of $0.155 \%$ over the full bandpass 4000-8000 $\AA$ (see upper dashed-dotted line in Fig. 4a). Such a value is significantly above what is observed. Therefore, the $\mathrm{TiO}$ abundance must be much lower than solar above altitudes corresponding to the observed AD level.

Assuming a constant mixing ratio, the best fit of the low resolution data set over the region 4000-5700 A gives a TiO abundance of $(8 . \pm 0.6) \times 10^{-4}$ solar. With this TiO abundance, the $\chi^{2}$ is reduced from 1754 to 1207 for $n=942$ degrees of freedom (using non rebinned spectra). However, this abundance cannot reproduce the $6250 \AA$ spectral feature and the flat absorption of the observations between $6200-8000 \AA$. We conclude that other absorbers must be considered to explain this part of the AD spectral profile.

\subsection{TiO and VO}

Since VO is the most important source of opacity after TiO (see Table 1), we add this molecule to our model. The chemistry of vanadium is quite similar to that of titanium, where the monoxide is in the gas phase at a temperature higher than the temperature of condensation.

In a first step, we consider a solar abundance over the whole atmosphere. As for TiO, VO with solar abundance cannot reproduce the observations, especially around $5000 \AA$. The abundance of VO must be lower. Assuming constant mixing ratios for both molecules, the best fit gives for TiO $(6 \pm 0.6) \times 10^{-4}$ and for VO $(3 \pm 0.5) \times 10^{-2}$ as shown in Fig. 4 b. We obtain a satisfactory fit with a $\chi^{2}$ of 817 , or a $\chi^{2} / n \approx 0.86$ in the low resolution data set. The continuous flat part of the AD curve can be well reproduce by absorption of $\mathrm{VO}$ molecules. The region around $5000 \AA$ already well reproduced with Rayleigh scattering and with sodium lines absorptions is not affected by the presence of $\mathrm{TiO}$ and $\mathrm{VO}$ molecules. However, the spectral features around $6250 \AA$ A remain unexplained assuming constant mixing ratios for both species. Note that sligthly lower abundances are necessary for $\mathrm{TiO}$ and $\mathrm{VO}$ if we consider the Knutson et al. (2007) results for the radii over $6000 \AA$.

\subsection{TiO, VO, and condensation}

With a large temperature gradient, $\mathrm{TiO}$ and $\mathrm{VO}$ abundances are expected to vary along the vertical atmospheric profile. Furthermore, the $P-T$ profile derived by Sing et al. (2008b) crosses the $\mathrm{TiO}$ and VO condensation curve (see Fig. 2). To check if a signature of condensation can be found in the present data set, we assume in this section that $\mathrm{TiO} / \mathrm{VO}$ molecules can be depleted above a given altitude. Indeed, $\mathrm{TiO}$ and $\mathrm{VO}$ are expected to condense into many $\mathrm{Ti}$ and $\mathrm{V}$-bearing compounds as described in detail by Lodders (2002). To implement this assumption, we introduce a maximum altitude, corresponding to an absorption depth of condensation ( $\left.\mathrm{AD}_{\text {cond }}\right)$, above which the absorption by the corresponding molecules in gas phase vanishes.

In the model, we derive the altitude of condensation from the intersection between the $P-T$ profile and the condensation curve. The condensation curves of $\mathrm{TiO}$ and VO depend mainly on the temperature (see Fig. 2 and Sharp \& Burrows 2007). The $\mathrm{TiO}$ condensation point occurs at a temperature of $1850 \mathrm{~K}$ and a pressure of 0.02 bar (see Fig. 2). This altitude corresponds to an $\mathrm{AD}$ of condensation $\mathrm{AD}_{\text {cond }}=0.0146 \%$. In the case of $\mathrm{VO}$, the condensation point is at $1600 \mathrm{~K}$ and $0.01 \mathrm{bar}$, leading to $\mathrm{AD}_{\text {cond }}=0.0147 \%$ (see condensation limits in Fig. $4 \mathrm{c}$ ). 


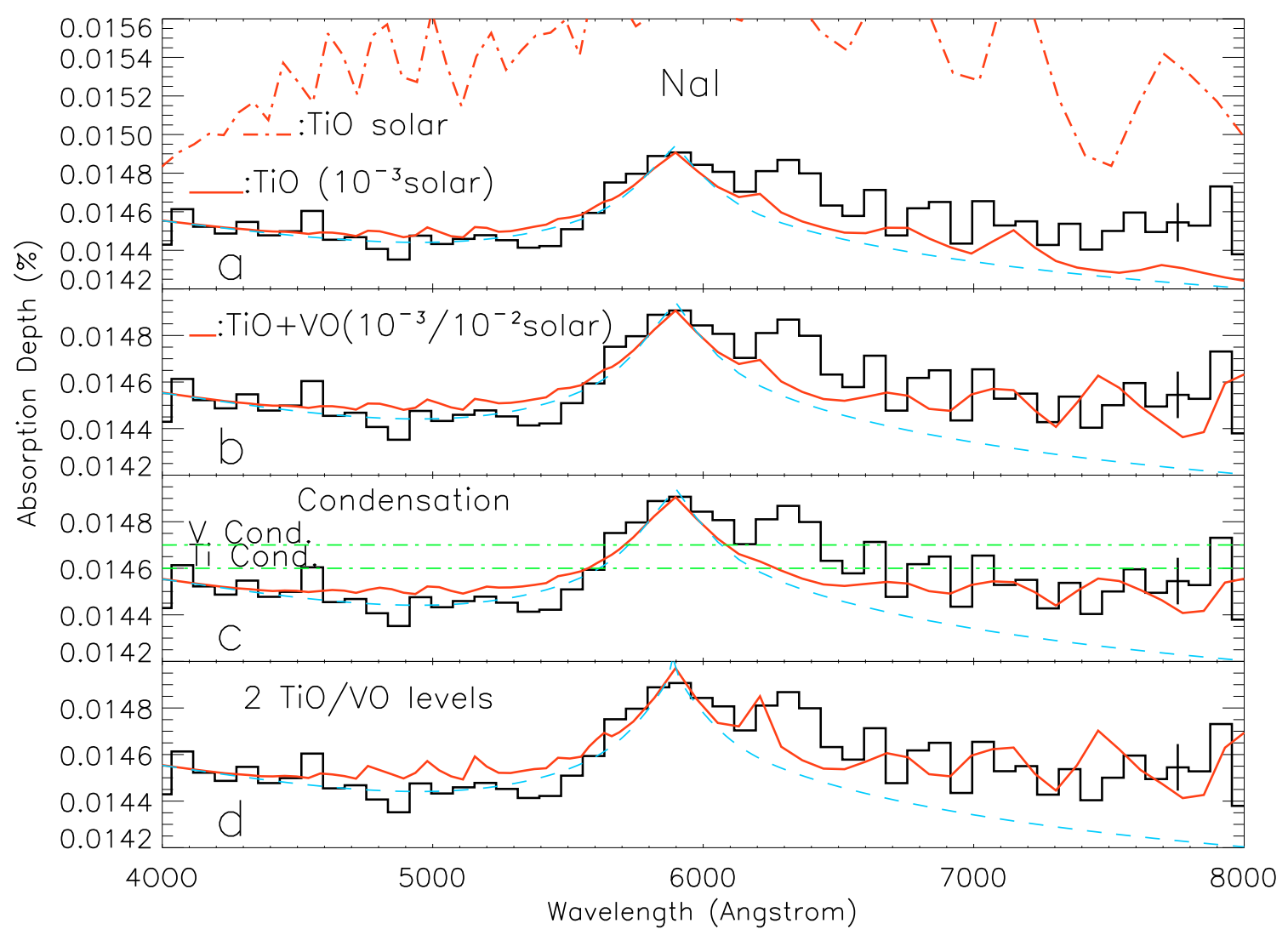

Fig. 4. Same as Fig. 1 with fits obtained with various models (continuous thick line). The observed $1 \sigma$ error bars are plotted around $7800 \AA$ (see also Fig. 10 of Sing et al. 2008). The dashed line shows the fit obtained using only the model with Rayleigh scattering and sodium absorption with the $T-P$ profile (Fig. 2). A remaining broad band absorption appears between 6200 to $8000 \AA$ which could be attributed to TiO and VO opacities. a) Model with a constant mixing ratio of TiO. A constant solar abundance of $\mathrm{TiO}$ is excluded (upper dashed-dotted line). The best fit gives an abundance of $(8 \pm 0.6) \times 10^{-4}$ solar (red solid line). b) Model with a constant mixing ratio of TiO and VO. Solar abundance for VO is also excluded. The red solid line is the best fit which includes $\mathrm{TiO}$ and $\mathrm{VO}$. c) $\mathrm{TiO}, \mathrm{VO}$, and condensation. The altitudes of condensation for titanium and for vanadium are both plotted (dash dotted lines) together with the best fit (red solid line). d) Best fit for two distinct levels containing TiO and VO (see Sect. 4.6). No clear solution emerges from the last three models (red solid line).

Using the $P-T$ profile presented in Fig. 2, TiO and $\mathrm{VO}$ $\mathrm{AD}_{\text {cond }}$ levels are lower than the $6250 \AA$ spectral feature $\mathrm{AD}$ peak. However, both $\mathrm{AD}_{\text {cond }}$ levels are found to be above the observed average $\mathrm{AD}$, above the continuous flat part between 6500-7500 $\AA$, and significantly above the lowest $\mathrm{AD}=$ $0.0144 \%$ around the left wing of the Na I at $5000 \AA$. Thus, these molecules cannot be in solar abundances below the altitude of condensation, otherwise the resulting $\mathrm{AD}$ curve would be at the level of the condensation limits. We fit the AD curve between $4000-8000 \AA$ and obtain an abundance for TiO and VO below their altitudes of condensation of $(8 \pm 0.5) \times 10^{-4}$ and $(6 \pm 0.4) \times$ $10^{-2}$ solar, respectively. We obtain a fit with a similar $\chi^{2}$ as before (Sect. 4.4) with one level of abundance for $\mathrm{TiO} / \mathrm{VO}$ over the whole atmosphere.

Although the "cold-trap" can explain the lowest observational ADs, our assumption of null abundances above this altitude cannot describe the observed $6250 \AA$ spectral signature. Thus TiO/VO molecules should remain in the upper part of the atmosphere if the feature is due to these species.

\subsection{TiO and VO in two separated layers}

In this section, we explore the possibility of the presence of $\mathrm{TiO}$ and VO molecules in the upper part of the atmosphere above the condensations altitudes. In the lower part of the atmosphere, we keep the abundances found in Sect. 4.5. Then a level of null abundance is imposed, corresponding to the condensation level. Finally another abundance level is chosen for the upper part of the atmosphere, where the temperature rises and again cross the condensation curves of $\mathrm{TiO} / \mathrm{VO}$. In the upper part of the atmosphere, where the pressure is lower, the $\mathrm{TiO}$ lines are less pressure broadened, numerous narrow peaks appear in the $\mathrm{TiO}$ opacity curve and, in particular, a strong peak appears at $6200 \AA$ (Fig. 4d).

The best fit gives a TiO and VO at respectively $(1 \pm 0.2) \times$ $10^{-4}$ and $(1 \pm 0.2) \times 10^{-2}$ solar abundances in the upper layer, above the altitudes of condensation. However the $\chi^{2}$ does not significantly decrease (802 for 944 degrees of freedom). The spectral features of the $\mathrm{AD}$ curve are now better fitted with $\mathrm{TiO}$, at least to first order, whereas VO molecules reproduce mainly the continuous flat part. In that way, we can more successfully reproduce the spectral feature, the continuous flat part and match the Na I blue wing simultaneously. Note that the flat part of the spectrum ratio cannot be reproduced if we choose a null abundance of $\mathrm{TiO} / \mathrm{VO}$ in the lower part of the atmosphere.

\section{Discussion}

We have highlighted that the HD 209458b atmosphere is optically thick at low pressures and this requires new absorbers 
Table 2. Bests $\chi_{2}$ fits performed for various models and the derived $\mathrm{TiO}$ and $\mathrm{VO}$ abundances.

\begin{tabular}{cccc}
\hline \hline Model & $\chi_{2}$ & $\mathrm{TiO}\left(\times 10^{-4}\right)$ & $\mathrm{V0}\left(\times 10^{-2}\right)$ \\
\hline Only TiO & 1207 & $(8 . \pm 0.6)$ & 0 \\
TiO and VO & 817 & $(6 . \pm 0.6)$ & $(3 . \pm 0.5)$ \\
TiO and VO with condensation & 816 & $(8 . \pm 0.5)$ & $(6 . \pm 0.4)$ \\
2 levels of TiO and VO & 802 & $(1 . \pm 0.2)$ & $(1 . \pm 0.2)$ \\
\hline
\end{tabular}

between $6000-8000 \AA$. The observation of the $\mathrm{Na}$ I in the atmosphere excludes the presence of clouds at pressures under 30 mbar in the observed limb of the planet. We have shown that Li I and K I absorption cannot reproduce the broad band absorptions observed. Nonetheless, we derive upper limits for their abundances at $2 \times 10^{-3}$ solar for K I and $2 \times 10^{-1}$ for Li I.

The Barman (2007) analysis of the Knuston et al. (2007) results and the Sing et al. (2008b) analysis both agree that remaining absorbants are required to explain the continuous broad band absorption observed between 6200 and $8000 \AA$, since the radii derived in both reductions are significantly above the model with no TiO nor VO molecules (see Fig. 1). As a consequence, the presence of remaining absorbants, possibly $\mathrm{TiO}$ and $\mathrm{VO}$, is mandatory to explain the observations. This result does not depend on the $P-T$ profile adopted. The abundances of $\mathrm{TiO}$ and VO are only poorly constrained by any $P-T$ profile. We decided to use the $P-T$ profile derived by Sing et al. (2008) as an example. In that case, the lower part of the $P-T$ profile, i.e. below the condensation curves, is the most critical part for the determination of the $\mathrm{TiO}$ and $\mathrm{VO}$ abundances. The abundances of $\mathrm{TiO}$ and VO molecules are mainly constrained by the level of the observed AD curve between 6500 and $8000 \AA$ and by the pressure of the lower point. Indeed, for this lower point, the $\mathrm{TiO}$ and VO lines are mostly pressure broadened. Thus, the abundances of these species are mainly sensitive to the pressure and not to the temperature in the lower part of this profile. The $P-T$ profile above the condensation curves mostly constrains the spectral features, especially the $6250 \AA$ one, as seen for the case two level of $\mathrm{TiO}$ and $\mathrm{VO}$. However, we do not detect any $\mathrm{TiO} / \mathrm{VO}$ narrow spectrale features, thus any $P-T$ profile above the condensation curve can be adopted. With the $P-T$ profile used in this study, we have shown that no clear solutions emerge. We cannot distinguish wether condensation should be taken into account, or if two levels of TiO and VO molecules should be considered (see Table 2). Thus, other profiles could led to the same conclusion for the presence of $\mathrm{TiO}$ and $\mathrm{VO}$ and/or of potential additional absorbers.

Although no typical $\mathrm{TiO}$ and $\mathrm{VO}$ spectral signatures have been identified unambiguously in the observed spectrum, we suggest that the opacities of those molecules are the best candidates to explain the remaining continuous broad band absorption observed between 6200 and $8000 \AA$. Theoretical absorption with models including $\mathrm{TiO} / \mathrm{VO}$ with abundances below solar were evaluated to match the data reasonably well. Using the $P-T$ profile from Sing et al. (2008b), we derived upper limits for the $\mathrm{TiO}$ and $\mathrm{VO}$ abundances. The model without $\mathrm{TiO}$ or with $\mathrm{TiO}$ but no VO molecules give the worst $\chi_{2}$ solutions. We found that the abundance of $\mathrm{TiO}$ should be around $10^{-4}$ to $10^{-3}$ solar, and the abundance VO around $10^{-3}$ to $10^{-2}$ solar. The fits become marginally worse on the blue side of the sodium wing when adding TiO and VO (see Fig. 4). This is because the $P-T$ profile used in our work was obtained by fitting the observation on the blue side of the sodium line with a model which contains only Rayleigh scattering and sodium opactity (Sing et al. 2008b) which helped limit the number of free parameters in the fit such that a convergent solution easily could be found. This model fits precisely the observed data for this part of the spectrum (see the dotted line in Figs. 1 and 4). The addition of the $\mathrm{TiO}$ and VO opacities in this model contributes to slightly decreasing the quality of the fit on the blue side of the sodium line (see continous line in Fig. 4). However, This is of marginal consequence for the $\mathrm{TiO}$ and $\mathrm{VO}$ evaluations in our study since these are mostly controlled by the main part of the observations in the redder range of the spectrum. Considering the quality of the observations, this does not affect the $\mathrm{TiO}$ and VO evaluations. Hence, the use of the $P-T$ profile derived by Sing et al. (2008) with only Rayleigh scattering and sodium opacity is sufficient to derive reasonably good estimates of the $\mathrm{TiO}$ and $\mathrm{VO}$ abundances.

Two layers of $\mathrm{TiO} / \mathrm{VO}$ in the atmosphere are better suited to explain the $6250 \AA$ spectral feature, however the $\chi^{2}$ is not significantly improved (Table 2). The $6250 \AA$ spectral feature is seen in the two independent spectra obtained at both low resolution, as mentioned here, and at medium resolution as observed by Sing et al. (2008a) in the G750M STIS spectra.

The tholins, polyacetylenes, or various non-equilibrium compounds at high-altitude could also be responsible for the temperature inversion. However, the study of these molecules requires a full non-equilibrium chemical model which is not the purpose of this work. TiO and VO molecules are very good high-altitude absorber candidates (Hubeny et al. 2003; Fortney et al. 2007). Fortney et al. (2008) highlighted the importance of gaseous $\mathrm{TiO}$ and $\mathrm{VO}$ opacity in their model of highly irradiated close-in giant planets. They define two classes of irradiated atmosphere: those that are warm enough to have a strong opacity due to $\mathrm{TiO}$ and VO gases ("pM Class" planets), and those that are cooler ("pL Class" planets), dominated by Na I and K I. Our possible detection of $\mathrm{TiO} / \mathrm{VO}$ and $\mathrm{Na} \mathrm{I}$ in the hot atmosphere of HD 209458b confirm that this planet is located in the transition region between the two classes defined by these authors. As observed here, due to the presence of TiO/VO, HD 209458b's atmosphere absorbs incident energy between $6500 \AA$ and $8000 \AA$. Consequently, this planet has a hot stratosphere (around $2500 \mathrm{~K}$ ) with a temperature inversion (Burrows et al. 2007c). As another consequence, because of the thermal emission of the energy trapped by the $\mathrm{TiO} / \mathrm{VO}$ absorption, the planet appears very bright during the mid infrared secondary eclipse (Knutson et al. 2008).

The first titanium condensates appearing at high temperature and high pressure are $\mathrm{Ti}_{3} \mathrm{O}_{5}$ and $\mathrm{CaTiO}_{3}$ (Burrows \& Sharp 1999; Lodders 2002). The condensation of vanadium starts at lower temperature $(1600 \mathrm{~K})$ than for titanium $(1800 \mathrm{~K})$. The vanadium condenses into solid $\mathrm{VO}$ and then into $\mathrm{V}_{2} \mathrm{O}_{3}$. Thus, depletion of $\mathrm{TiO}$ should start at a lower altitude than for VO. Below $1600 \mathrm{~K}, \mathrm{TiO}, \mathrm{VO}$ and major refractory elements are absent, leaving monoatomic $\mathrm{NaI}$ and $\mathrm{KI}$ to dominate the spectrum. Nevertheless, sodium and potassium chlorides become increasingly abundant with decreasing temperature, especially for $\mathrm{KCl}$ which is the dominant $\mathrm{K}$-bearing compound. Condensed potassium depletes the atmosphere of atomic KI, as seen with $\mathrm{Na}$ I, leading to reduced signatures. This could explain why $\mathrm{Na}$ I is seen in abundance in the low resolution data, but not $\mathrm{KI}$. In the lower warmer atmosphere, where wide atomic $\mathrm{K}$ line wings would be observable even at low resolution, $\mathrm{TiO}$ and VO likely make up the surrounding continuum, further masking the signature.

An alternative $P-T$ profile with $\mathrm{Na}$ ionization has been also proposed (Sing et al. 2008b). A model with $\mathrm{Na}$ ionization 
leaves a wide range of temperatures possible in the middle atmosphere. Within the framework of equilibrium chemistry, this profile presents a lower temperature gradient (Fortney et al. 2003). The minimum temperature is below the $\mathrm{TiO} / \mathrm{VO}$ condensation curves, and above the sodium condensation curve. We did not enter into a detailed study of $\mathrm{TiO} / \mathrm{VO}$ abundances with these $P-T$ profile; however, we note that these profiles would have increase the abundance of $\mathrm{TiO}$ and VO molecules in the middle atmosphere. See Sing et al. (2008b) for more details about ionization.

If present in the upper part of the atmosphere, TiO/VO molecules are not fully depleted by condensation. Thermochemical equilibrium calculations with rainout (Burrows \& Sharp 1999; Lodders 2002; Hubeny et al. 2003) have shown that $\mathrm{TiO}$ and $\mathrm{VO}$ can exist at high- $T /$ low- $P$ points such as in the upper part of our $P-T$ profile. The presence of $\mathrm{TiO}$ and $\mathrm{VO}$ at high- $T /$ high- $P$ points leads to a situation in which there is two levels of $\mathrm{TiO} / \mathrm{VO}$. This was proposed by Hubeny et al. (2003) to explain the presence of a temperature inversion in strongly irradiated planet atmospheres. Since the $P-T$ profile used here twice crosses the $\mathrm{Ti}$ and $\mathrm{V}$ condensation curves, two levels containing $\mathrm{TiO} / \mathrm{VO}$ molecules in gas phase could be separated by a middle level that is free of those molecules, as proposed by Hubeny et al. (2003). Nonetheless, a cold trap region is usually expected to deplete the upper low- $P$ region. Flushing out the upper atmosphere of $\mathrm{TiO} / \mathrm{VO}$ would rule out $6250 \AA$ feature as being due to TiO. However, for now, it has not been proven that the cold-trap effect would deplete those molecules, especially for the hydrodynamically escaping type of upper atmosphere (Vidal-Madjar et al. 2003, 2004, 2008; Lecavelier 2004; Lecavelier et al. 2007; Ehrenreich et al. 2008).

New observations with higher signal-to-noise ratio and better resolution, together with improved chemical models, are required to address $\mathrm{TiO}$ and $\mathrm{VO}$ spectral absorption features and detect specific band heads.

Acknowledgements. We thank our anonymous referee and our editor for their comments that strengthened the presentation of our results. We also thank Adam Burrows, Jonathan Fortney and Mark Marley for useful discussions.

\section{References}

Allard, F., Hauschildt, P. H., Alexander, D. R., Tamanai, A., \& Schweitzer, A. 2001, ApJ, 556, 357

Allard, N. F., Allard, F., \& Kielkopf, J. F. 2005, A\&A, 440, 1195

Ballester, G. E., Sing, D. K., \& Herbert, F. 2007, Nature, 445, 511

Barman, T. 2007, ApJ, 661, L191

Brown, T. M. 2001, ApJ, 553, 1006

Burrows, A., \& Sharp, C. M. 1999, ApJ, 512, 843

Burrows, A., \& Volobuyev, M. 2003, ApJ, 583, 985
Burrows, A., Marley, M. S., \& Sharp, C. M. 2000, ApJ, 531, 438

Burrows, A., Burgasser, A. J., Kirkpatrick, J. D., et al. 2002, ApJ, 573, 394

Burrows, A., Sudarsky, D., \& Hubeny, I. 2006, ApJ, 650, 1140

Burrows, A., Hubeny, I., Budaj, J., Knutson, H. A., \& Charbonneau, D. 2007, ApJ, 668, L171

Burrows, A., Budaj, J., \& Hubeny, I. 2008, ApJ, 678, 1436

Charbonneau, D., Brown, T. M., Noyes, R. W., \& Gilliland, R. L. 2002, ApJ, 568,377

Charbonneau, D., Allen, L. E., Megeath, S. T., et al. 2005, ApJ, 626, 523

Ehrenreich, D., Tinetti, G., Lecavelier des Etangs, A., Vidal-Madjar, A., \& Selsis, F. 2006, A\&A, 448, 379

Ehrenreich, D., Lecavelier des Etangs, A., Hébrard, G., et al. 2008, A\&A, 483, 933

Fegley, B. J., \& Lodders, K. 1996, ApJ, 472, L37

Fortney, J. J., Sudarsky, D., Hubeny, I., et al. 2003, ApJ, 589, 615

Fortney, J. J., Cooper, C. S., Showman, A. P., Marley, M. S., \& Freedman, R. S. 2006, ApJ, 652, 746

Fortney, J. J., Lodders, K., Marley, M. S., \& Freedman, R. S. 2008, ApJ, 678, 1419

Hubbard, W. B., Fortney, J. J., Lunine, J. I., et al. 2001, ApJ, 560, 413

Hubeny, I., Burrows, A., \& Sudarsky, D. 2003, ApJ, 594, 1011

Iro, N., Bézard, B., \& Guillot, T. 2005, A\&A, 436, 719

Knutson, H. A., Charbonneau, D., Noyes, R. W., Brown, T. M., \& Gilliland, R. L. 2007, ApJ, 655, 564

Knutson, H. A., Charbonneau, D., Allen, L. E., Burrows, A., \& Megeath, S. T. 2008, ApJ, 673, 526

Kirkpatrick, J. D. 2005, ARA\&A, 43, 195

Lecavelier des Etangs, A. 2007, A\&A, 461, 1185

Lecavelier des Etangs, A., Vidal-Madjar, A., McConnell, J. C., \& Hébrard, G. 2004, A\&A, 418, L1

Lecavelier des Etangs, A., Pont, F., Vidal-Madjar, A., \& Sing, D. 2008a, A\&A, 481, L83

Lecavelier des Etangs, A., Vidal-Madjar, A., Désert, J.-M., \& Sing, D. 2008b, A\&A, 485, 865

Lodders, K. 1999, ApJ, 519, 793

Lodders, K. 2002, ApJ, 577, 974

Lodders, K., \& Fegley, B., Jr. 2006, Astrophysics Update 2, 1

Morton, D. C. 1991, ApJS, 77, 119

Plez, B. 1998, A\&A, 337, 500

Pont, F., Knutson, H., Gilliland, R. L., Moutou, C., \& Charbonneau, D. 2008, MNRAS, 385, 109

Redfield, S., Endl, M., Cochran, W. D., \& Koesterke, L. 2008, ApJ, 673, L87

Rowe, J. F., Matthews, J. M., Seager, S., et al. 2006, ApJ, 646, 1241

Sauval, A. J., \& Tatum, J. B. ApJS, 56, 193

Sharp, C. M., \& Burrows, A. 2007, ApJS, 168, 140

Seager, S., \& Sasselov, D. D. 1998, ApJ, 502, L157

Seager, S., \& Sasselov, D. D. 2000, ApJ, 537, 916

Sing, D. K., Vidal-Madjar, A., Désert, J.-M., Lecavelier des Etangs, A., \& Ballester, G. 2008a, ApJ, 686, 658

Sing, D. K., Vidal-Madjar, A., Lecavelier des Etangs, A., et al. 2008b, ApJ, 686, 667

Snellen, I. A. G., Albrecht, S., de Mooij, E. J. W., \& Le Poole, R. S. 2008, A\&A, 487, 357

Sudarsky, D., Burrows, A., \& Pinto, P. 2000, ApJ, 538, 885

Sudarsky, D., Burrows, A., \& Hubeny, I. 2003, ApJ, 588, 1121

Vidal-Madjar, A., Lecavelier des Etangs, A., Désert, J.-M., et al. 2003, Nature, 422,143

Vidal-Madjar, A., Désert, J.-M., Lecavelier des Etangs, A., et al. 2004, ApJ, 604, L69

Vidal-Madjar, A., Lecavelier des Etangs, A., Désert, J.-M., et al. 2008, ApJ, 676, L57 